TAIWANESE JOURNAL OF MATHEMATICS

Vol. 13, No. 5, pp. 1609-1622, October 2009

This paper is available online at http://www.tjm.nsysu.edu.tw/

\title{
AN INTERPOLATION THEOREM RELATED TO THE HARDY SPACE WITH NON-DOUBLING MEASURE
}

\author{
Guoen $\mathrm{Hu}^{1}$, Jiali Lian and Huoxiong $\mathrm{Wu}^{2, *}$
}

\begin{abstract}
Let $\mu$ be a nonnegative Radon measure satisfying the growth condition that $\mu(B(x, r)) \leq C r^{n}$ for any $x \in \mathbb{R}^{d}$ and $r>0$ and some fixed positive constants $C$ and $n$ with $0<n \leq d$. Let $H_{\text {atb }}^{1, \infty}(\mu)$ be the Hardy space associated with $\mu$ which was introduced by Tolsa. In this paper, a new interpolation theorems related to $H_{\mathrm{atb}}^{1, \infty}(\mu)$ is established and the interpolation theorem of Tolsa is improved.
\end{abstract}

\section{INTRODUCTION}

During the last decade, considerable attention has been paid to the study of function spaces and boundedness of operators on these space (see [1-9]). Let $\mu$ be a nonnegative Radon measure on $\mathbb{R}^{d}$ which only satisfies the following growth condition: there exist constants $C_{0}>0$ and $n \in(0, d]$ such that for all $x \in \mathbb{R}^{d}$ and $r>0$,

$$
\mu(B(x, r)) \leq C_{0} r^{n},
$$

where $B(x, r)$ is the open ball centered at some point $x \in \mathbb{R}^{d}$ and having radius $r$. The measure $\mu$ in (1.1) is not assumed to satisfy the doubling condition which is a key assumption in the analysis on spaces of homogeneous type. We recall that $\mu$ is said to satisfy the doubling condition if there exists some constant $C>0$ such that $\mu(B(x, 2 r)) \leq C \mu(B(x, r))$ for all $x \in \mathbb{R}^{d}$ and $r>0$. Some important non-doubling measures as in (1.1) and the motivation for developing the analysis related to such measures can be found in [9], see also [4]. We only point out

Received November 3, 2007, accepted January 23, 2008.

Communicated by Yongsheng Han.

2000 Mathematics Subject Classification: 42B25, 42B30.

Key words and phrases: Interpolation, Hardy space, Non-doubling measure, Maximal function.

${ }^{1}$ Partially supported by the NNSF of China (No. 10671210).

${ }^{2}$ Corresponding author, partially supported by the NNSF of China (No. 10571122, 10771054).

*Corresponding author. 
that the analysis with non-doubling measures plays an essential role in solving the long-standing open Painlevé's problem by Tolsa in [8].

In his remarkable work [6], Tolsa found a suitable substitute for the classical BMO space when the underlying measure satisfies $(1.1), \operatorname{RBMO}(\mu)$. The space $\operatorname{RBMO}(\mu)$ enjoys the properties which are parallel to those of the space $\operatorname{BMO}\left(\mathbb{R}^{d}\right)$, for example, $\operatorname{RBMO}(\mu)$ is big enough so that a $L^{2}(\mu)$ bounded Calderon-Zygmund operator is also bounded from $L^{\infty}(\mu)$ to $\operatorname{RBMO}(\mu)$, and small enough to satisfy the properties (such as John-Nirenberg inequality) of the classical BMO space. Also, Tolsa established the following interpolation theorem (see [6, p. 131]).

Theorem 1. Let $T$ be a linear operator which is bounded from $H_{\mathrm{atb}}^{1, \infty}(\mu)$ to $L^{1}(\mu)$, and bounded from $L^{\infty}(\mu)$ to $\operatorname{RBMO}(\mu)$. Then for any $p \in(1, \infty), T$ extends boundedly to $L^{p}(\mu)$, where $H_{\mathrm{atb}}^{1, \infty}(\mu)$ is the atomic Hardy space with the measure $\mu$ in (1.1), see Definition 1 below.

The main purpose of this paper is to establish a new interpolation theorem related to $H_{\mathrm{atb}}^{1, \infty}(\mu)$ which improves Tolsa's interpolation theorem above. To states our main results, we first give some definitions and notation.

By a cube $Q \subset \mathbb{R}^{d}$ we mean a closed cube with sides parallel to the axes and centered at some point of $\operatorname{supp} \mu$. We denote its side length by $l(Q)$. Given $\alpha>1$ and $\beta>\alpha^{n}$, we say that $Q$ is $(\alpha, \beta)$-doubling if $\mu(\alpha Q) \leq \beta \mu(Q)$, where $\alpha Q$ is the cube concentric with $Q$ with side length $\alpha l(Q)$. It was pointed by Tolsa in [5] that there are a lot of "big" doubling cubes. To be precise, given any point $x \in \operatorname{supp}(\mu)$ and $c>0$, there exists some $(\alpha, \beta)$-doubling cube $Q$ centered at $x$ with $l(Q) \geq c$ due to the growth condition (1.1). On the other hand, if $\beta>\alpha^{d}$, then for $\mu$-a.e. $x \in \mathbb{R}^{d}$, there exists a sequence of $(\alpha, \beta)$-doubling cubes $\left\{Q_{i}\right\}_{i \in \mathbb{N}}$ centered at $x$ with $l\left(Q_{i}\right) \rightarrow 0$ as $i \rightarrow \infty$. In what follows, for definiteness, if $\alpha$ and $\beta$ are not specified, by a doubling cube we mean $\left(2,2^{d+1}\right)$-doubling cube. Given two cubes $Q_{1} \subset Q_{2}$, set

$$
K_{Q_{1}, Q_{2}}=1+\sum_{k=1}^{N_{Q_{1}, Q_{2}}} \frac{\mu\left(2^{k} Q_{1}\right)}{\left[l\left(2^{k} Q_{1}\right)\right]^{n}},
$$

where $N_{Q_{1}, Q_{2}}$ is the first positive integer $k$ such that $l\left(2^{k} Q_{1}\right) \geq l\left(Q_{2}\right)$; see [6] for some basic properties of $K_{Q_{1}, Q_{2}}$.

Given a cube $Q \subset \mathbb{R}^{d}$, let $\widetilde{Q}$ be the smallest doubling cube in the sequence $\left\{2^{k} Q\right\}_{k \geq 0}$, and by $m_{Q}(f)$ the mean value of $f$ on $Q$, namely,

$$
m_{Q}(f)=\frac{1}{\mu(Q)} \int_{Q} f(x) d \mu(x) .
$$

The sharp maximal operator associated with the measure $\mu$ in (1.1) is defined by 


$$
M^{\sharp} f(x)=\sup _{Q \ni x} \frac{1}{\mu\left(\frac{3}{2} Q\right)} \int_{Q}\left|f(y)-m_{\widetilde{Q}} f\right| d \mu(y)+\sup _{\substack{R \supset Q \ni x \\ Q, R \text { doubling }}} \frac{\left|m_{Q} f-m_{R} f\right|}{K_{Q, R}} .
$$

Definition 1. Let $\rho>1$ and $1<p \leq \infty$. A function $b \in L_{\text {loc }}^{1}(\mu)$ is called a $p$-atomic block if

(1) there exists some cube $R$ such that $\operatorname{supp} b \subset R$,

(2) $\int_{\mathbb{R}^{d}} b(x) d \mu(x)=0$,

(3) for $j=1,2$, there are functions $a_{j}$ supported on cubes $Q_{j} \subset R$ and numbers $\lambda_{j} \in \mathbb{R}$ such that $b=\lambda_{1} a_{1}+\lambda_{2} a_{2}$, and

$$
\left\|a_{j}\right\|_{L^{p}(\mu)} \leq\left[\mu\left(\rho Q_{j}\right)^{1-1 / p} K_{Q_{j}, R}\right]^{-1} .
$$

Then we define

$$
|b|_{H_{a t b}^{1, p}(\mu)}=\left|\lambda_{1}\right|+\left|\lambda_{2}\right| .
$$

We say that $f \in H_{a t b}^{1, p}(\mu)$ if there are $p$-atomic blocks $\left\{b_{i}\right\}_{i \in \mathbb{N}}$ such that

$$
f=\sum_{i=1}^{\infty} b_{i}
$$

with $\sum_{i=1}^{\infty}\left|b_{i}\right|_{H_{a t b}^{1, p}(\mu)}<\infty$. The $H_{a t b}^{1, p}(\mu)$ norm of $f$ is defined by

$$
\|f\|_{H_{a t b}^{1, p}(\mu)}=\inf \left\{\sum_{i}\left|b_{i}\right|_{H_{a t b}^{1, p}(\mu)}\right\}
$$

where the infimum is taken over all the possible decompositions of $f$ in atomic blocks.

The space $H_{\mathrm{atb}}^{1, \infty}(\mu)$ was introduced by Tolsa in [6], and further considered by Tolsa in [7]. Moreover, it was proved by Tolsa in [6, 7] that the definition of $H_{a t b}^{1, p}(\mu)$ is independent of the chosen constant $\rho>1$. Moreover, for any $p \in$ $(1, \infty)$

$$
H_{\mathrm{atb}}^{1, p}(\mu)=H_{\mathrm{atb}}^{1, \infty}(\mu)
$$

with equivalent norms.

Our main result can be stated as follows.

Theorem 2. Let $T$ be an operator which satisfies that

(i) $\left|T f_{1}-T f_{2}\right| \leq\left|T\left(f_{1}-f_{2}\right)\right|$; 
(ii) there is another operator $T_{1}$, which is bounded from $L^{p_{0}}(\mu)$ to $L^{q_{0}, \infty}(\mu)$ for some $p_{0}, q_{0}$ with $p_{0} \leq q_{0}$ and $p_{0}, q_{0} \in(1, \infty]$ such that for any bounded function $f$ with compact support,

$$
M^{\sharp}(T f)(x) \leq C T_{1} f(x) ;
$$

(iii) for some $q_{1} \in[1, \infty), T$ is bounded from $H_{\mathrm{atb}}^{1, \infty}(\mu)$ to $L^{q_{1}, \infty}(\mu)$, that is, there is a constant $C>0$, such that for any $\lambda>0$ and any $f \in H_{\text {atb }}^{1, \infty}(\mu)$,

$$
\mu\left(\left\{x \in \mathbb{R}^{d}:|T f(x)|>\lambda\right\}\right) \leq C\left(\lambda^{-1}\|f\|_{H_{\text {atb }}^{1, \infty}(\mu)}\right)^{q_{1}} .
$$

Then for any $p, q \in(1, \infty)$ with

$$
\frac{1}{p}=t+\frac{1-t}{p_{0}}, \frac{1}{q}=\frac{t}{q_{1}}+\frac{1-t}{q_{0}}, \quad t \in(0,1),
$$

$T$ is bounded from $L^{p}(\mu)$ to $L^{q}(\mu)$.

\section{Proof of Theorem 2}

We begin with the John-Stromberg sharp maximal operator with a measure in (1.1), which was introduced in [1]. For a cube $Q$ with $\mu(Q) \neq 0$, and a real-valued locally integrable function $f, m_{f}(Q)$, the median value of $f$ on the cube $Q$, is defined to be one of numbers such that

$$
\mu\left(\left\{y \in Q: f(y)>m_{f}(Q)\right\}\right) \leq \frac{1}{2} \mu(Q)
$$

and

$$
\mu\left(\left\{y \in Q: f(y)<m_{f}(Q)\right\}\right) \leq \frac{1}{2} \mu(Q) .
$$

For the case that $\mu(Q)=0$, we set $m_{f}(Q)=0$ for any real-valued locally integrable function $f$. If $f$ is complex-valued, the median value of $f$ is defined by $m_{f}(Q)=$ $m_{\operatorname{Re}(f)}(Q)+i m_{\operatorname{Im}(f)}(Q)$, where $i^{2}=-1$.

Let $s \in\left(0,2^{-d-2}\right)$. For each fixed cube $Q$ and a locally integrable function $f$, define $m_{0, s ; Q}(f)$ by

$$
\begin{aligned}
& m_{0, s ; Q}(f)=\inf \left\{t>0: \mu(\{y \in Q:|f(y)|>t\})<s \mu\left(\frac{3}{2} Q\right)\right\} \\
& \text { when } \mu(Q) \neq 0,
\end{aligned}
$$

and $m_{0, s ; Q}(f)=0$ when $\mu(Q)=0$. The John-Strömberg maximal operator $M_{0, s}$, and the doubling John-Stromberg maximal operator $M_{0, s}^{d}$, associated with measure in (1.1) are defined by 


$$
M_{0, s} f(x)=\sup _{Q \ni x} m_{0, s ; Q}(f), \quad M_{0, s}^{d} f(x)=\sup _{\substack{Q \ni x \\ Q \text { doubling }}} m_{0, s ; Q}(f),
$$

and the John-Strömberg sharp maximal operator $M_{0, s}^{\sharp}$ is defined by

$$
M_{0, s}^{\sharp} f(x)=\sup _{Q \ni x} m_{0, s ; Q}\left(f-m_{f}(\widetilde{Q})\right)+\sup _{\substack{R \supset Q \ni x \\ Q, R \text { doubling }}} \frac{\left|m_{f}(Q)-m_{f}(R)\right|}{K_{Q, R}} .
$$

We then have

Lemma 1. Let $0<s<2^{-d-2}$. Then for any locally integrable function $f$ and any $\lambda>0$,

(i) $\left\{x \in \mathbb{R}^{d}:|f(x)|>\lambda\right\} \subset\left\{x \in \mathbb{R}^{d}: M_{0, s}^{d} f(x) \geq \lambda\right\} \cup \Theta$ with $\mu(\Theta)=0$;

(ii)

$$
\mu\left(\left\{x \in \mathbb{R}^{d}: M_{0, s} f(x)>\lambda\right\}\right) \leq C s^{-1} \mu\left(\left\{x \in \mathbb{R}^{d}:|f(x)|>\lambda\right\}\right),
$$

where $C>0$ is a constant depending on $d$.

Proof. This lemma was essentially proved in [1]. For the sake of self-contained, we present the proof here. Let $M^{d}$ be the maximal operator defined by

$$
M^{d} f(x)=\sup _{\substack{Q \ni x \\ Q \text { doubling }}} \frac{1}{\mu(Q)} \int_{Q}|f(y)| d \mu(y) .
$$

By the Lebesgue differential lemma, we know that for $\mu$ almost $x \in \mathbb{R}^{d}$,

$$
|f(x)| \leq M^{d} f(x)
$$

and so

$$
\begin{aligned}
\left\{x \in \mathbb{R}^{d}:|f(x)|>\lambda\right\} & =\left\{x \in \mathbb{R}^{d}: \chi_{\left\{y \in \mathbb{R}^{d}:|f(y)|>\lambda\right\}}(x)=1\right\} \\
& \subset\left\{x \in \mathbb{R}^{d}: M^{d}\left(\chi_{\left\{y \in \mathbb{R}^{d}:|f(y)|>\lambda\right\}}\right)(x)>s 2^{d+1}\right\} \cup \Theta .
\end{aligned}
$$

On the other hand, a straightforward computation leads to that

$$
\left\{x \in \mathbb{R}^{d}: M^{d}\left(\chi_{\left\{y \in \mathbb{R}^{d}:|f(y)|>\lambda\right\}}\right)(x)>s 2^{d+1}\right\} \subset\left\{x \in \mathbb{R}^{d}: M_{0, s}^{d} f(x) \geq \lambda\right\} .
$$

The conclusion (i) then follows directly.

To prove (ii), for each fixed $\lambda>0$ and $r>0$, set

$$
M_{0, s}^{r} f(x)=\sup _{Q \ni x, l(Q)<r} m_{0, s ; Q}(f)
$$


and

$$
E_{r, \lambda}=\left\{x \in \mathbb{R}^{d}: M_{0, s}^{r} f(x)>\lambda\right\} .
$$

For any fixed $x \in E_{r, \lambda}$, there is a cube $Q_{x}$ containing $x$ and $l\left(Q_{x}\right)<r$, such that

$$
\mu\left(\left\{y \in Q_{x}:|f(y)|>\lambda\right\}\right) \geq s \mu\left(\frac{3}{2} Q_{x}\right) .
$$

Applying the Besicovitch covering lemma, we can select $N$ family of cubes $\left\{Q_{j}^{k}\right\}_{1 \leq j \leq N, k \in \Lambda_{j}}$ from $\left\{Q_{x}\right\}_{x \in E_{r, \lambda}}$, such that

(a)

$$
E_{r, \lambda} \subset \bigcup_{j=1}^{N} \bigcup_{k \in \Lambda_{j}} \frac{3}{2} Q_{j}^{k}
$$

(b) there is a constant $C>0$ such that for any fixed $j, 1 \leq j \leq k$,

$$
\sum_{k \in \Lambda_{j}} \chi_{Q_{j}^{k}} \leq C
$$

where $N$ is the Besicovitch constant. It then follows that

$$
\begin{aligned}
\mu\left(E_{r, \lambda}\right) & \leq \sum_{j=1}^{N} \sum_{k \in \Lambda_{j}} \mu\left(\frac{3}{2} Q_{j}^{k}\right) \\
& \leq s^{-1} \sum_{j=1}^{N} \sum_{k \in \Lambda_{j}} \mu\left(\left\{y \in Q_{j}^{k}:|f(y)|>\lambda\right\}\right) \\
& \leq C s^{-1} \mu\left(\left\{y \in \mathbb{R}^{d}:|f(y)|>\lambda\right\}\right) .
\end{aligned}
$$

Letting $r \rightarrow \infty$ then leads to our desired conclusion.

To prove Theorem 2, we also need some preliminary lemmas.

Lemma 2. Let $s \in\left(0,2^{-d-2}\right)$ and $T$ be an operator which satisfies that

$$
\left|T f_{1}(x)-T f_{2}(x)\right| \leq\left|T\left(f_{1}-f_{2}\right)(x)\right| .
$$

There is a constant $C>0$ such that for any $f_{1}$ and $f_{2}$,

$$
M_{0, s}^{\sharp}\left[T\left(f_{1}+f_{2}\right)\right](x) \leq C M^{\sharp}\left(T f_{1}\right)(x)+C M_{0, s / 2}\left(T f_{2}\right)(x) .
$$


Proof. For any cube $Q$, a straightforward computation yields

$$
\begin{aligned}
m_{0, s ; Q}\left(T\left(f_{1}+f_{2}\right)-m_{T\left(f_{1}+f_{2}\right)}(\widetilde{Q})\right) \leq & m_{0, s / 2 ; Q}\left(T f_{1}-m_{T f_{1}}(\widetilde{Q})\right) \\
& +m_{0, s / 2 ; Q}\left(T\left(f_{1}+f_{2}\right)-T f_{1}\right) \\
& +\left|m_{T\left(f_{1}+f_{2}\right)}(\widetilde{Q})-m_{T f_{1}}(\widetilde{Q})\right|
\end{aligned}
$$

Note that for any cube $I$, locally integrable function $h$ and constant $c, m_{h}(I)-c$ is a median value of $h-c$ on $I$, namely,

$$
m_{h}(I)-c=m_{h-c}(I)
$$

Thus,

$$
\begin{aligned}
\left|m_{T\left(f_{1}+f_{2}\right)}(\widetilde{Q})-m_{T f_{1}}(\widetilde{Q})\right| \leq & \left|m_{T\left(f_{1}+f_{2}\right)-m_{T f_{1}}(\widetilde{Q})}(\widetilde{Q})\right| \\
\leq & 2 m_{0, s ; \widetilde{Q}}\left(T\left(f_{1}+f_{2}\right)-m_{T f_{1}}(\widetilde{Q})\right) \\
\leq & 2 m_{0, s / 2 ; \widetilde{Q}}\left(T f_{1}-m_{T f_{1}}(\widetilde{Q})\right) \\
& +2 m_{0, s / 2 ; \widetilde{Q}}\left(T\left(f_{1}+f_{2}\right)-T f_{1}\right)
\end{aligned}
$$

where the second inequality follows from the fact that for any doubling cube $I$, locally integrable function $h$ and $s \in\left(0,2^{-d-2}\right)$,

$$
\left|m_{h}(I)\right| \leq 2 m_{0, s ; I}(h),
$$

see [1, Lemma 2.5]. This in turn leads to that

$$
\begin{aligned}
m_{0, s ; Q}\left(T\left(f_{1}+f_{2}\right)-m_{T\left(f_{1}+f_{2}\right)}(\widetilde{Q})\right) \leq & 3 \inf _{x \in Q} M_{0, s / 2}^{\sharp}\left(T f_{1}\right)(x) \\
& +3 \inf _{x \in Q} M_{0, s / 2}\left(T f_{2}\right)(x) .
\end{aligned}
$$

On the other hand, we can verify that for any two doubling cubes $Q \subset R$,

$$
\begin{aligned}
\left|m_{T\left(f_{1}+f_{2}\right)}(Q)-m_{T\left(f_{1}+f_{2}\right)}(R)\right| \leq & \left|m_{T\left(f_{1}+f_{2}\right)}(Q)-m_{T f_{1}}(Q)\right| \\
& +\left|m_{T\left(f_{1}+f_{2}\right)}(R)-m_{T f_{1}}(R)\right| \\
& +\left|m_{T f_{1}}(Q)-m_{T f_{1}}(R)\right| \\
\leq & 2 m_{0, s / 2 ; Q}\left(T f_{1}-m_{T f_{1}}(Q)\right) \\
& +2 m_{0, s / 2 ; Q}\left(T\left(f_{1}+f_{2}\right)-T f_{1}\right)
\end{aligned}
$$




$$
\begin{aligned}
& \quad+2 m_{0, s / 2 ; R}\left(T f_{1}-m_{T f_{1}}(R)\right) \\
& +2 m_{0, s / 2 ; R}\left(T\left(f_{1}+f_{2}\right)-T f_{1}\right) \\
& \quad+\left|m_{T f_{1}}(Q)-m_{T f_{1}}(R)\right| \\
& \leq 4 \inf _{x \in Q} M_{0, s / 2}^{\sharp}\left(T f_{1}\right)(x)+4 \inf _{x \in Q} M_{0, s / 2}\left(T f_{2}\right)(x) \\
& \quad+\left|m_{T f_{1}}(Q)-m_{T f_{1}}(R)\right| .
\end{aligned}
$$

We then get that

$$
M_{0, s}^{\sharp}\left[T\left(f_{1}+f_{2}\right)\right](x) \leq C M_{0, s / 2}^{\sharp}\left(T f_{1}\right)(x)+C M_{0, s / 2}\left(T f_{2}\right)(x) .
$$

Therefore, the proof of the estimate (2.4) can be reduced to proving that

$$
M_{0, s}^{\sharp} h(x) \leq C M^{\sharp} h(x) .
$$

Let $M^{\natural}$ be the sharp maximal operator defined by

$$
\begin{aligned}
M^{\natural} f(x)= & \sup _{Q \ni x} \frac{1}{\mu\left(\frac{3}{2} Q\right)} \int_{Q}\left|f(y)-m_{f}(\widetilde{Q})\right| d \mu(y) \\
& +\sup _{\substack{R \supset Q \ni x \\
Q, R \text { doubling }}} \frac{\left|m_{f}(Q)-m_{f}(R)\right|}{K_{Q, R}} .
\end{aligned}
$$

Observe that for any cube $Q$

$$
m_{0, s / 2 ; Q}\left(f-m_{f}(\widetilde{Q})\right) \leq \frac{2}{s \mu\left(\frac{3}{2} Q\right)} \int_{Q}\left|f(y)-m_{f}(\widetilde{Q})\right| d \mu(y) .
$$

It then follows that

$$
M_{0, s / 2}^{\sharp} f(x) \leq 2 s^{-1} M^{\natural} f(x) .
$$

Recall that for any cube $I$,

$$
\frac{1}{\mu(I)} \int_{I}\left|f(y)-m_{f}(I)\right| d \mu(y) \leq \frac{1}{\mu(I)} \int_{I}\left|f(y)-m_{I}(f)\right| d \mu(y)
$$

(see [5, p. 115]). It then follows that

$$
\begin{aligned}
\frac{1}{\mu\left(\frac{3}{2} Q\right)} \int_{Q}\left|f(y)-m_{f}(\widetilde{Q})\right| d \mu(y) \leq & \frac{1}{\mu\left(\frac{3}{2} Q\right)} \int_{Q}\left|f(y)-m_{\widetilde{Q}}(f)\right| d \mu(y) \\
& +\frac{1}{\mu(\widetilde{Q})} \int_{\widetilde{Q}}\left|f(y)-m_{f}(\widetilde{Q})\right| d \mu(y) \\
\leq & C \inf _{x \in Q} M^{\sharp} f(x) .
\end{aligned}
$$


On the other hand, for any two doubling cubes $Q$ and $R$, with $Q \subset R$,

$$
\begin{aligned}
\left|m_{f}(Q)-m_{f}(R)\right| \leq & \left|m_{Q}(f)-m_{f}(Q)\right|+\left|m_{R}(f)-m_{f}(R)\right|+\left|m_{Q}(f)-m_{R}(f)\right| \\
\leq & \frac{1}{\mu(Q)} \int_{Q}\left|f(y)-m_{f}(Q)\right| d \mu(y) \\
& +\frac{1}{\mu(R)} \int_{R}\left|f(y)-m_{f}(R)\right| d \mu(y)+\left|m_{Q}(f)-m_{R}(f)\right| \\
\leq & C K_{Q}, R \inf _{x \in Q} M^{\sharp} f(x) .
\end{aligned}
$$

Combining the last two estimates leads to that

$$
M^{\natural} f(x) \leq C M^{\sharp} f(x),
$$

and

$$
\begin{aligned}
M_{0, s}^{\sharp}\left[T\left(f_{1}+f_{2}\right)\right](x) & \leq C M_{0, \frac{s}{2}}^{\sharp}\left(T f_{1}\right)(x)+C M_{0, \frac{s}{2}}\left(T f_{2}\right)(x) \\
& \leq 2 s^{-1} C M^{\natural}\left(T f_{1}\right)(x)+C M_{0, \frac{s}{2}}\left(T f_{2}\right)(x) \\
& \leq C M^{\sharp}\left(T f_{1}\right)(x)+C M_{0, \frac{s}{2}}\left(T f_{2}\right)(x),
\end{aligned}
$$

then completes the proof of Lemma 2.

Lemma 3. Let $T, T_{1}, T_{2}$ be three operators such that for any $x \in \mathbb{R}^{d}$,

$$
\left|T\left(f_{1}+f_{2}\right)(x)\right| \leq\left|T_{1} f_{1}(x)\right|+\left|T_{2} f_{2}(x)\right| .
$$

Suppose that

(i) for $p_{0}, q_{0}$ with $p_{0} \leq q_{0}$ and $p_{0}, q_{0} \in(1, \infty]$, $T_{1}$ is bounded from $L^{p_{0}}(\mu)$ to $L^{q_{0}, \infty}(\mu)$, when $q_{0}=\infty, L^{q_{0}, \infty}(\mu)$ should be replaced by $L^{q_{0}}(\mu)$;

(ii) for some $q_{1} \in[1, \infty), T_{2}$ is bounded from $H_{\mathrm{atb}}^{1, \infty}(\mu)$ to $L^{q_{1}, \infty}(\mu)$.

Then for any $p, q$ with

$$
\frac{1}{p}=t+\frac{1-t}{p_{0}}, \frac{1}{q}=\frac{t}{q_{1}}+\frac{1-t}{q_{0}}, t \in(0,1),
$$

$T$ is bounded from $L^{p}(\mu)$ to $L^{q, \infty}(\mu)$.

Proof. Our goal is to prove that there is a constant $C>0$ such that for any $\lambda>0$, and bounded function $f$ with compact support,

$$
\lambda^{q} \mu\left(\left\{x \in \mathbb{R}^{d}:|T f(x)|>\lambda\right\}\right) \leq C\left(\int_{\mathbb{R}^{d}}|f(x)|^{p} d \mu(x)\right)^{q / p} .
$$


By homogeneity, we may assume that $\|f\|_{L^{p}(\mu)}=1$. For each fixed $\lambda>0$ and bounded function $f$ with compact, observe that if $\|\mu\|<\infty$ and $\lambda^{q / p} \leq$ $\|f\|_{L^{1}(\mu)} /\|\mu\|$, the inequality (2.6) follows directly, since by the Hölder inequality,

$$
\lambda^{q} \mu\left(\left\{x \in \mathbb{R}^{d}:|T f(x)|>\lambda\right\}\right) \leq C\|f\|_{L^{1}(\mu)}^{p} \leq C .
$$

Thus, we may assume $\|\mu\|=\infty$, or $\|\mu\|<\infty$ and $\lambda^{q / p}>\|f\|_{L^{1}(\mu)} /\|\mu\|$. Note that

It then follows that

$$
\frac{\frac{1}{q}-\frac{1}{q_{0}}}{\frac{1}{q_{1}}-\frac{1}{q_{0}}}=\frac{\frac{1}{p}-\frac{1}{p_{0}}}{1-\frac{1}{p_{0}}}, \frac{\frac{1}{q_{1}}-\frac{1}{q}}{\frac{1}{q_{1}}-\frac{1}{q_{0}}}=\frac{1-\frac{1}{p}}{1-\frac{1}{p_{0}}} .
$$

$$
\frac{\frac{1}{q}-\frac{1}{q_{0}}}{\frac{1}{q_{1}}-\frac{1}{q}}=\frac{\frac{1}{p}-\frac{1}{p_{0}}}{1-\frac{1}{p}}
$$

and so

$$
\frac{\left(q_{0}-q\right) p_{0}}{\left(p_{0}-p\right) q_{0}}=\frac{q-q_{1}}{(p-1) q_{1}} .
$$

Let $\theta=\left(q-q_{1}\right) /(p-1) q_{1}$. Applying the Caldeon-Zygmund decomposition to $|f|^{p}$ at level $\lambda^{\theta p}$ (see [6, p. 131-132]), we know that there exist a sequences of cubes $\left\{Q_{j}\right\}_{j}$ such that

(a) the cubes $\left\{Q_{j}\right\}_{j}$ have bounded overlaps, that is, there is a constant $C$ such that $\sum_{j} \chi_{Q_{j}}(x) \leq C$;

(b) $\frac{1}{\mu\left(2 Q_{j}\right)} \int_{Q_{j}}|f(x)|^{p} d \mu(x)>\frac{\lambda^{\theta p}}{2^{d+1}}$;

(c) for any $\eta>0, \frac{1}{\mu\left(2 \eta Q_{j}\right)} \int_{\eta Q_{j}}|f(x)|^{p} d \mu(x) \leq \frac{\lambda^{\theta p}}{2^{d+1}}$;

(d) $|f(x)| \leq \lambda^{\theta}$, $\mu$-a. e. $x \in \mathbb{R}^{d} \backslash \cup_{j} Q_{j}$;

(e) for each fixed $j$, let $R_{j}$ be the smallest $\left(6,6^{n+1}\right)$-doubling cube of the form $6^{k} Q_{j}$ for $k \in \mathbb{N}$. Set $w_{j}=\chi_{Q_{j}} / \sum_{k \geq 1} \chi_{Q_{k}}(x)$. Then there is a function $\phi_{j}$ with supp $\phi_{j} \subset R_{j}$ and some positive constant $C$ satisfying

$$
\int_{\mathbb{R}^{d}} \phi_{j}(x) d \mu(x)=\int_{Q_{j}} f(x) w_{j}(x) d \mu(x), \sum_{j}\left|\phi_{j}(x)\right| \leq C \lambda^{\theta},
$$

and

$$
\left(\int_{R_{j}}\left|\phi_{j}(x)\right|^{p} d \mu(x)\right)^{1 / p}\left[\mu\left(R_{j}\right)\right]^{1 / p^{\prime}} \leq \frac{C}{\lambda^{\theta(p-1)}} \int_{Q_{j}}|f(x)|^{p} d \mu(x) .
$$


We can decompose $f$ as

$$
f(x)=g(x)+b(x)
$$

where

$$
g(x)=f(x) \chi_{\mathbb{R}^{d} \backslash \cup_{j} Q_{j}}(x)+\sum_{j} \phi_{j}(x)
$$

and

$$
b(x)=\sum_{j}\left(f(x) w_{j}(x)-\phi_{j}(x)\right) .
$$

It is easy to verify that

$$
\|g\|_{L^{p_{0}}}^{p_{0}} \leq\|g\|_{L^{\infty}(\mu)}^{p_{0}-p}\|g\|_{L^{p}(\mu)}^{p} \leq C \lambda^{\theta\left(p_{0}-p\right)}
$$

and

$$
\|b\|_{H_{\text {atb }}^{1, p}(\mu)} \leq C \lambda^{-\theta(p-1)} .
$$

This in turn leads to that

$\mu\left(\left\{x \in \mathbb{R}^{d}:\left|T_{1} g(x)\right|>\lambda / 2\right\}\right) \leq C \lambda^{-q_{0}}\|g\|_{L^{p_{0}(\mu)}}^{q_{0}} \leq C \lambda^{-q_{0}} \lambda^{\theta\left(p_{0}-p\right) q_{0} / p_{0}} \leq C \lambda^{-q}$.

and

$$
\mu\left(\left\{x \in \mathbb{R}^{d}:\left|T_{2} b(x)\right|>\lambda / 2\right\}\right) \leq C \lambda^{-q_{1}}\|b\|_{H_{\mathrm{atb}}^{1, p}(\mu)}^{q_{1}} \leq C \lambda^{-q} .
$$

then

$$
\mu\left(\left\{x \in \mathbb{R}^{d}:|T f(x)|>\lambda\right\}\right) \leq C \lambda^{-q} .
$$

This completes the proof of Lemma 3.

Lemma 4. (see [1]). Let $s_{1} \in\left(0,2^{-d-2}\right)$ and $p \in(0, \infty)$. There is a constant $C_{1} \in(0,1)$ depending on $s_{1}$ such that for any $s_{2} \in\left(0, C_{1} s_{1}\right)$,

(i) if $\|\mu\|=\infty, f \in L^{p_{0}, \infty}(\mu)$ with $p_{0} \in[1, \infty)$ and

$$
\sup _{0<\lambda<R} \lambda^{p} \mu\left(\left\{x \in \mathbb{R}^{d}:|f(x)|>\lambda\right\}\right)<\infty
$$

for any $R>0$, then

$$
\sup _{\lambda>0} \lambda^{p} \mu\left(\left\{x \in \mathbb{R}^{d}: M_{0, s_{1}}^{d} f(x)>\lambda\right\}\right) \leq C \sup _{\lambda>0} \lambda^{p} \mu\left(\left\{x \in \mathbb{R}^{d}: M_{0, s_{2}}^{\sharp} f(x)>\lambda\right\}\right) ;
$$

(ii) if $\|\mu\|<\infty$ and $f \in L^{p_{0}, \infty}(\mu)$ with $p_{0} \in[1, \infty)$, then

$$
\begin{aligned}
& \sup _{\lambda>0} \lambda^{p} \mu\left(\left\{x \in \mathbb{R}^{d}: M_{0, s_{1}}^{d} f(x)>\lambda\right\}\right) \\
& \leq C \sup _{\lambda>0} \lambda^{p} \mu\left(\left\{x \in \mathbb{R}^{d}: M_{0, s_{2}}^{\sharp} f(x)>\lambda\right\}\right) \\
& \left.\quad+\|\mu\|\left(s_{1}\|\mu\|\right)\right)^{-p / p_{0}}\|f\|_{L^{p_{0}, \infty}(\mu)}^{p} .
\end{aligned}
$$


Proof of Theorem 2. At first, for any $s \in\left(0,2^{-d-2}\right)$, our assumption (ii) along with the estimate (2.5) tells us that the operator $M_{0, s}^{\sharp} \circ T$ is bounded from $L^{p_{0}}(\mu)$ to $L^{q_{0}, \infty}(\mu)$. On the other hand, the assumption (iii) in Theorem 2 via Lemma 1 (ii) states that $M_{0, s} \circ T$ is bounded from $H_{\text {atb }}^{1, \infty}(\mu)$ to $L^{q_{1}, \infty}(\mu)$. Therefore, it follows from Lemma 2 and Lemma 3 that $M_{0, s}^{\sharp} \circ T$ is bounded from $L^{p}(\mu)$ to $L^{q, \infty}(\mu)$, that is, for any fixed $\lambda>0$ and bounded function $f$ with compact support,

$$
\lambda^{q} \mu\left(\left\{x \in \mathbb{R}^{d}: M_{0, s}^{\sharp} T f(x)>\lambda\right\}\right) \leq C\|f\|_{L^{p}(\mu)}^{q}
$$

provided that $p$ and $q$ satisfies

$$
\frac{1}{p}=t+\frac{1-t}{p_{0}}, \frac{1}{q}=\frac{t}{q_{1}}+\frac{1-t}{q_{0}}, t \in(0,1) .
$$

We can now conclude the proof of Theorem 2. Set

$$
L_{0,0}^{\infty}(\mu)=\left\{f: f \text { is bounded, has compact support, } \int_{\mathbb{R}^{d}} f(x) d \mu(x)=0\right\} .
$$

It is well known that $L_{0,0}^{\infty}(\mu)$ is a density subset of $L^{p}(\mu)$ for any $p \in[1, \infty)$. For each fixed $f \in L_{0,0}^{\infty}(\mu)$, which implies $f \in H_{a t b}^{1, \infty}(\mu)$, our hypothesis guarantee that $T f \in L^{q_{1}, \infty}(\mu)$ and so for any $R>0, q>q_{1}$,

$\sup _{0<\lambda<R} \lambda^{q} \mu\left(\left\{x \in \mathbb{R}^{d}:|T f(x)|>\lambda\right\}\right) \leq R^{q-q_{1}} \sup _{\lambda>0} \lambda^{q_{1}} \mu\left(\left\{x \in \mathbb{R}^{d}:|T f(x)|>\lambda\right\}\right)<\infty$.

By the standard density argument, we need only to prove Theorem 2 for $f \in L_{0,0}^{\infty}(\mu)$ in the following two cases:

Case 1. $\|\mu\|=\infty$. By Lemma 1, Lemma 4 (i) and (2.7), we have

$$
\begin{aligned}
\sup _{\lambda>0} \lambda^{q} \mu\left(\left\{x \in \mathbb{R}^{d}:|T f(x)|>\lambda\right\}\right) & \leq \sup _{\lambda>0} \lambda^{q} \mu\left(\left\{x \in \mathbb{R}^{d}: M_{0, s_{1}}^{d} T f(x) \geq \lambda\right\}\right) \\
& \leq C \sup _{\lambda>0} \lambda^{q} \mu\left(\left\{x \in \mathbb{R}^{d}: M_{0, s_{2}}^{\sharp} T f(x) \geq \lambda\right\}\right) \\
& \leq C\|f\|_{L^{p}(\mu)}^{q} .
\end{aligned}
$$

This implies $T$ is bounded from $L^{p}(\mu)$ to $L^{q, \infty}(\mu)$. On the other hand, the assumption (i) implies that $T$ is sublinear. Thus, by Marcinkiewicz's interpolation theorem, we know that $T$ is also bounded from $L^{p}(\mu)$ to $L^{q}(\mu)$.

Case 2. $\|\mu\|<\infty$. By a trivial computation, we see that for each fixed $p \in$ $(1, \infty)$

$$
\|f\|_{H_{\mathrm{atb}}^{1, p}(\mu)} \leq C\|\mu\|^{1-1 / p}\|f\|_{L^{p}(\mu)} .
$$


By Lemma 1 (i), Lemma 4 (ii) and (2.7), we have

$$
\begin{aligned}
& \sup _{\lambda>0} \lambda^{q} \mu\left(\left\{x \in \mathbb{R}^{d}:|T f(x)|>\lambda\right\}\right) \\
\leq & \sup _{\lambda>0} \lambda^{q} \mu\left(\left\{x \in \mathbb{R}^{d}: M_{0, s_{1}}^{d} T f(x) \geq \lambda\right\}\right) \\
\leq & C \sup _{\lambda>0} \lambda^{q} \mu\left(\left\{x \in \mathbb{R}^{d}: M_{0, s_{2}}^{\sharp} T f(x) \geq \lambda\right\}\right)+\|\mu\|\left(s_{1}\|\mu\|\right)^{-\frac{q}{q_{1}}}\|T f\|_{L^{q_{1}, \infty}(\mu)}^{q} \\
\leq & C\|f\|_{L^{p}(\mu)}^{q}+C s_{1}^{-\frac{q}{q_{1}}}\|\mu\|^{1-\frac{q}{q_{1}}}\|f\|_{H_{\text {atb }}^{1, \infty}(\mu)}^{q} \\
\leq & C\|f\|_{L^{p}(\mu)}^{q},
\end{aligned}
$$

which together with the same arguments as in Case 1 implies that $T$ is bounded from $L^{p}(\mu)$ to $L^{q}(\mu)$. This completes the proof of Theorem 2 .

\section{REFERENCES}

1. G. Hu and D. Yang, Weighted norm inequalities for maximal singular integral operators with non doubling measures, Submitted.

2. J. Mateu, P. Mattila, A. Nicolau and J. Orobitg, BMO for nondoubling measures, Duke Math. J., 102 (2000), 533-565.

3. F. Nazarov, S. Treil and A. Volberg, Cauchy integral and Calderon-Zygmund operators on nonhomogeneous spaces, Internat. Math. Res. Notices, 15 (1997), 703-726.

4. F. Nazarov, S. Treil and A. Volberg, Weak type estimates and Cotlar inequalities for Calderon-Zygmund operators on nonhomogeneous spaces, Internat. Math. Res. Notices, 9 (1998), 463-487.

5. X. Tolsa, A proof of weak $(1,1)$ inequality for singular integrals with non doubling measures based on a Calderon-Zygmund decomposition, Publ. Mat., 45 (2001), 163-174.

6. X. Tolsa, BMO, $H^{1}$ and Calderon-Zygmund operators for non doubling measures, Math. Ann., 319 (2001), 89-149.

7. X. Tolsa, The space $H^{1}$ for nondoubling measures in terms of a grand maximal operator, Trans. Amer. Math. Soc., 355 (2003), 315-348.

8. X. Tolsa, Painleve's problem and semiadditivity of analytic capacity, Acta Math., 190 (2003), 105-149.

9. J. Verdera, The fall of the doubling condition in Calderon-Zygmund theory, Publ. Mat., Extra (2002), 275-292. 


\section{Guoen $\mathrm{Hu}$}

Department of Applied Mathematics,

Zhengzhou Imformation Science and Technology Institute,

P. O. Box 1001-747,

Zhengzhou 450002,

P. R. China

E-mail: guoenxx@yahoo.com.cn

\section{Jiali Lian}

Department of Computer Science and Engineering,

Wuyi University,

Wuyi, Fujian 354300,

P. R. China

E-mail: jiali-lian@sina.com

Huoxiong Wu

School of Mathematical Sciences,

Xiamen University,

Xiamen Fujian 361005,

P. R. China

E-mail: huoxwu@xmu.edu.cn 